\title{
BARRERAS PARA EL MANEJO EFECTIVO DEL DOLOR CRÓNICO
}

\section{CONSTANZA LONDOÑO ${ }^{1}$, FRANCOISE CONTRERAS ${ }^{2}$, GABRIEL DELGADILLO ${ }^{1}$, SERGIO TOBÓN ${ }^{3}$ y STEFANO VINACCIA ${ }^{4}$}

\author{
${ }^{1}$ Universidad Nacional de Colombia \\ ${ }^{2}$ Universidad Santo Tomás, Colombia \\ ${ }^{3}$ Universidad de Antioquia, Colombia \\ ${ }^{4}$ Universidad de San Buenaventura, Colombia
}

\begin{abstract}
El propósito de este articulo es presentar una revisión teórica acerca de los principales factores involucrados en el manejo del efectivo del dolor crónico, los cuales giran en torno a aspectos teóricos, formas de evaluación, tratamiento, aspectos psicológicos y relación cuidador/paciente. Se plantea la necesidad de implementar evaluaciones y tratamientos integrales, resultado de la intervención transdisciplinar, que incluya pacientes, familiares y cuidadores, para alcanzar mayores niveles de efectividad y favorezca la calidad de vid del paciente.
\end{abstract}

Palabras clave: Dolor crónico, calidad de vida.

\section{Barriers for the effective management of chronic pain}

The purpose of this article was to present a theoretical revision concerning the principal factors involved in effective management of chronic pain, which are based around theoretical aspects, forms of evaluation, treatments, psychological aspects, and the caretaker- patient relation. The necessity to implement integrated evaluation and treatment is established, resulting in trandisciplinary intervention which included patients, family member and caregivers to achieve better levels of effective and enhance the patient's quality of life.

Key words: Chronic Pain, quality of life.

El dolor es una de las principales causas de sufrimiento y discapacidad en el mundo, y constituye un problema de salud pública con importantes repercusiones sociales y económicas. Actualmente con el incremento en la esperanza de vida, aumenta la probabilidad de padecer enfermedades crónicas, cuya característica fundamental es que son prolongadas, pues aunque sean mortales, causan la muerte solo tras un periodo largo de tiempo (Brannon y Feist, 2001). Este tipo de enfermedades suelen estar acompañadas de procesos dolorosos de intensidad variable, cuya fisiología no está bien definida (a diferencia del dolor agudo), incluso es un tipo de dolor que ha llegado a

Correspondencia: Constanza Londoño, Departamento de Psicología, Universidad Nacional de Colombia, Bogotá, Colombia. Correo-e: gatatpsi@hotmail.com considerarse como desadaptativo y sin propósito aparente (Allende y Lara, 2000; Harkins et al., 1989).

Entre este tipo de enfermedades, están las cefaleas, artritis, trastornos gastrointestinales y cáncer entre otros, en este ultimo caso, se presentan niveles significativos de dolor, sobre todo en estados avanzados de la enfermedad (Bonica et al., 1983), cuya intensidad varia según el tipo de cáncer presentado y las diversas fuentes causantes de dolor en este tipo de pacientes (Cruzado y Labrador 1991; Stratton, 1996).

Los pacientes crónicos, sufren detrimento en su calidad de vida debido a que suelen presentar dolor permanente, discapacidad, alteraciones del sueño, disminución del apetito, cambios en el esta- 
do de animo, decrementos en el rendimiento y la productividad laboral e incluso, dificultades familiares y sociales (Stratton, 1996), estudios epidemiológicos han demostrado que estos pacientes presentan altos niveles de distrés psicosocial.

A pesar de los grandes avances médicos que aportan a la evaluación y tratamiento de la mayoría de enfermedades, es evidente que en el tema del dolor existen aún muchos aspectos que requieren ser estudiados, pues su manejo es complicado y en ocasiones resulta poco efectivo. Las barreras para el control eficaz del dolor están típicamente relacionadas con dificultades teórico-practicas, evaluativos, asociadas al tratamiento, a las características del paciente y de su relación con el cuidador. A continuación se expondrán brevemente cada una de ellas.

\section{DIFICULTADES TEÓRICO-PRACTICAS}

Inicialmente el dolor era explicado dentro de un contexto de estímulo respuesta (Eisenberg, 1994) como un evento causado de forma lineal. Sin embargo, desde 1986, este concepto fue sustituido por el de la Asociación Internacional para el Estudio del Dolor (IASP) que lo definió como una experiencia sensorial y emocional displacentera, asociada al daño del tejido, real o potencial o descrita en términos de tal daño. Ésta definición puso de manifiesto el componente subjetivo y experiencial en la percepción del dolor, ya no como una sensación primaria sino como un proceso complejo en el que incluso, el daño tisular puede o no existir en un momento dado.

Teóricamente, en la actualidad se asume que el dolor debe ser entendido bajo un modelo multidisciplinario, dado que no solo la esfera sensorial está implicada, sino que en él se conjugan factores físi- cos, psicológicos, sociales y ambientales (Simón, 1999). Los modelos multidimensionales de dolor que parten de la definición de la IASP incluye tres dimensiones básicas del dolor; (1) la sensurial, que engloba la acción de mecanismos neurofisiológicos que discrimina, identifica, describe la intensidad del dolor y lo ubica espacial y temporalmente, (2) la motivacional-afectiva que caracteriza al dolor como desagradable y desencadena la reacción de escape o de acercamiento, y (3) la cognitivo evaluativa que se ocupa de la atención, sugestión, evaluación, creencias y valores sobre el dolor; y afecta de manera importante las otras dimensiones.

Como se puede observar, en teoría se ha superado la visión simplista del fenómeno del dolor, sin embargo, estos avances no han sido del todo llevados a la práctica. En la mayoría de los casos, el dolor se sigue tratando como una afección física y sólo en contadas ocasiones, se ofrece un tratamiento integral que incluya las dimensiones motivacional y evaluativa. Por otra parte, muchos de los modelos multidisciplinares propuestos, tardan años en ser comprendidos y llevados a la práctica en ambientes hospitalarios, debido a su lenta difusión y a que algunos administradores de la salud piensan que pueden llegar a ser muy costosos.

\section{DIFICULTADES ASOCIADAS AL PROCESO DE EVALUACIÓN}

El hecho de que el dolor sea percibido únicamente por el individuo que lo padece y que en el, interactúen de forma compleja diversos factores, implica una serie de problemas metodológicos en su medición y evaluación. Concretamente, la subjetividad del reporte de dolor supone dificultad para su evaluación, pues no todos los pacientes que padecen una mis- 
ma afección lo presentan, y es muy difícil llegar a predecir quien lo experimentara para llevar a cabo procedimientos psicoprofilácticos integrales, lo que implica que en la actualidad, el dolor solo pueda ser abordado a nivel asistencial y no de manera preventiva.

Por otra parte, los instrumentos de autoinforme diseñados para evaluar el dolor, aunque son de suma utilidad para propósitos clínicos y de investigación, presentan dificultades en la precisión de las medidas obtenidas y deben ser validados exhaustivamente para proveer mediciones estandarizadas y confiables de las variables psicológicas que influyen en la percepción y comportamiento del dolor.

Para evaluar el dolor se han diseñado diversas escalas y cuestionarios, entre ellas están la Escala de Puntuación de Intensidad del Dolor (EPID), la Escala y Visual Análoga del Dolor (VAPS), el McGill Pain Questionnaire (MPQ), el Illness Behavior Questionnaire (IBQ), el Pain Behavior Check List (PBCL), y el West Haven-Yale Multidimensional Pain Inventory, entre otras (Turk y Melzack, 1992).

Dado que el fenómeno del dolor es evidentemente un proceso complejo, su evaluación debe incluir mecanismos fisiopatológicos, factores causales, intensidad del dolor, patrón de presentación (agudo, crónico), antecedentes relacionados con el pronóstico (alcoholismo y trastorno mental), impacto del dolor en la actividad cotidiana, relaciones familiares y estado de animo del paciente (Stratton, 1996), factores que en la mayoría de los casos no se considera, debido probablemente a la carencia de equipos multidisciplinares en los servicios de salud, a la falta de entrenamiento o a la escasez de presupuesto. Esta situación tiene que ver con las dificultades anteriormente expuestas, en teoría se asumen las distintas dimensiones del dolor, pero en la práctica, su evaluación no las incluye de manera integrada.

\section{DIFICULTADES ASOCIADAS AL TRATAMIENTO}

Teniendo en cuenta las consideraciones anteriormente expuestas, actualmente se plantea que el tratamiento, al igual que la evaluación del dolor, debe hacerse de manera transdisciplinar, y que en el deben participar diversos profesionales de la salud (médicos, psicólogos, enfermeras, terapistas ocupacionales y fisioterapeutas entre otros), atendiendo a los aspectos físicos, psicológicos, sociales, vocacionales y funcionales. En países en vías de desarrollo, esta condición por lo general no se cumple, debido a problemas de presupuesto y de formación., lo que hace que el tratamiento para abordar el dolor sea poco efectivo.

Estudios han evidenciado que solo la implementación de técnicas integrales, han demostrado ser efectivas para modificar la magnitud del dolor percibido en pacientes crónicos (Lee et al., 2002; Maconi et al., 2003; Harkins et al., 1989). Particularmente, se ha determinado que la terapia cognitiva combinada con terapia farmacológica genera menor costo postratamiento (consultas por dolor, cirugías y procedimientos anestésicos) que la aplicación de terapias exclusivamente farmacológicas (Cipher et al., 2001). Se reconoce ampliamente, la importancia de incluir la terapia cognitivo-conductual como parte del tratamiento del dolor, en términos de costos, mejora en la capacidad funcional, mejora en la calidad de vida del paciente y disminución del consumo de servicios de salud. En general la intervención psicológica debe orientarse hacia el incremento de la creencia de control del paciente ante la situación, y el desarrollo de habilidades específicas para afrontar la enfermedad y los síntomas. 
Otro aspecto importante con respecto al tratamiento del dolor es que aunque la organización mundial de la salud ha planteado una escala farmacológica de tres pasos para la dosificación y prescripción de analgésicos de acuerdo con el curso del dolor, existe una marcada tendencia a recetar en casos de dolor moderado, fármacos recomendados para dolor severo (Herrera y Orazi, 2002).

De acuerdo con dichos parámetros, el paso 1 se recomienda para dolor leve (no opiáceos), el 2 para dolor moderado (combinación de bajas dosis de opiáceos y no opiáceos) y el 3 para dolor severo (opiáceos). Las barreras sociales y culturales relacionadas con el miedo a la adicción. a la tolerancia a los opiáceos, a los efectos secundarios, a ser juzgado como adicto por solicitarlos, o a que se puedan tomar medidas disciplinarias, especialmente en países donde la ley regula drásticamente la acción médica (Gross et al., 2003), suelen constituir barreras importantes para el tratamiento del dolor crónico (Schumacher et al., 2002). Tales temores, en ocasiones poco fundamentados, suelen provenir de la pobre comunicación entre el paciente y los profesionales de la salud, de la dificultad para el acceso a información adecuada y oportuna y la insuficiencia en información y adiestramiento de los profesionales de la salud (Willoughby et al., 2002).

Finalmente, la intervención suele llevarse a cabo sin la participación activa del paciente, lo que genera en el, baja percepción de control. Se hace necesario por tanto, desarrollar programas de entrenamiento en el manejo y tratamiento del dolor, dirigidos a personal sanitario, a pacientes y a familiares, para que éstos asuman un papel activo dentro de la terapia y contribuyan a mejorar su eficacia en el alivio (Baron, 1993), incidiendo favorablemente en la calidad de vida $y$ adaptación del paciente y su familia (Hadedorrn et al., 2002).

\section{DIFICULTADES ASOCIADAS A LAS CARACTERÍSTICAS PSICOLÓGICAS DEL PACIENTE}

La depresión, ansiedad, limitaciones en la movilidad, creencias en torno a la enfermedad, convicción de carencia de control sobre el dolor y percepción de falta de apoyo social, afectan la respuesta al tratamiento en algunos pacientes con dolor crónico. De hecho se ha identificado una clara asociación entre dolor intenso y la tendencia marcada a la catastrofización (Jacobsen et al., 2004) que tiene serias implicaciones tanto en la adherencia al tratamiento como en el curso de la enfermedad.

La depresión suele presentarse con frecuencia en pacientes con altos niveles de dolor, y con el incremento en el uso de drogas analgésicas (Ciaramella y Paollo, 2001). El percibir un dolor mas severo está asociado con el detrimento en la calidad de vida, representada en la satisfacción actual y futura del paciente, lo que a su vez afecta la percepción de alivio (Hwang et al., 2002) y determina el grado de adherencia al tratamiento.

Los estilos de afrontamiento y la capacidad de adaptación del paciente también han sido estudiados. Las personas que asumen estilos adaptativos tienden a mostrar bajas respuestas punitivas ante el dolor y menos angustia que aquellas que utilizan estrategias disfuncionales, las cuales se asocian con bajo control vital percibido y una alta necesidad de soporte que los puede llevar a sobrecargar a sus cuidadores y afectar el éxito del tratamiento (Zaza et al., 2000).

Se ha demostrado igualmente una relación entre sensación/intensidad del dolor y respuestas de frustración, ira, agotamiento, invalidez e inutilidad en pacientes con dolor crónico. Esto representa una grave barrera tanto para la adherencia al tratamiento analgésico como para la conservación de la actividad diaria, pues el 
paciente centra toda su atención en la expresión de conductas de dolor. Sela et al. (2002) encontraron en pacientes con cáncer avanzado que éstos tienen mayor probabilidad de alcanzar altos niveles de dolor (entre 79 y 100 puntos en la escala de intensidad), asociados principalmente, a la frustración y al agotamiento.

\section{DIFICULTADES ASOCIADAS A LA RELACIÓN CUIDADOR - PACIENTE}

Las discrepancias entre las creencias que tiene el cuidador y el paciente sobre la autoeficacia para manejar el dolor y otros síntomas, constituye una dificultad asociada al manejo del dolor crónico, lo cual a su vez se ha relacionado con la pobre comunicación entre el paciente y su familia, y de la familia con los profesionales de la salud.

Se observa además que altos niveles de fatiga en cuidadores y altos niveles de síntomas en los pacientes, asociado a bajos puntajes en satisfacción con la relación, están asociados con la tendencia de los cuidadores a sobreestimar la autoeficacia del paciente. La incongruencia en la percepción de autoeficacia entre pacientes y cuidadores está asociada a su vez, con altos niveles de angustia en ambos, ya que esta situación puede llevar a que el paciente reciba menos soporte y asistencia de la que verdaderamente requiere (Porter et al., 2002).

Se debe tener en cuenta que los cuidadores se ven enfrentados a múltiples demandas entre las que se encuentran además de las labores diarias, el monitorear los síntomas, administrar medicamentos, lidiar con los efectos colaterales, comunicarse con el medico y otros profesionales (Porter et al., 2002). Estos altos niveles de estrés y fatiga a los que están sometidos los cuidadores, aumentan la probabilidad de cometer errores en la dosificación de los medicamentos u olvi- dar suministrarlos, lo que se relaciona con el abuso de los recursos sanitarios (Simón, 1999). Por todo ello, se hace necesario estudiar con mayor detenimiento los factores que promueven la educación de los cuidadores y su posibilidad de evaluar exitosamente el dolor del paciente para ofrecer el apoyo necesario de acuerdo con sus necesidades (Redinbaugh et al., 2002).

\section{CONCLUSIONES}

Dado el incremento de las enfermedades crónicas y los procesos dolorosos asociados, se hace necesario aumentar la investigación y el estudio para el manejo efectivo del dolor, de tal manera que se pueda incidir favorablemente en la calidad de vida de estas personas.

El tratamiento efectivo del dolor crónico implica asumir que éste es un proceso complejo que incluye y afecta aspectos fisiológicos, psicológicos y socioculturales por tanto, un tratamiento efectivo, implicaría llevar a la práctica los modelos teóricos existentes, validarlos y ajustarlos, de acuerdo con las características particulares de la población, lo cual requeriría de equipos transdisciplinares para su implementación.

Se hace necesario optimizar los instrumentos de evaluación, para que éstos incluyan, sino todos, la mayoría de los aspectos relacionados con la experiencia dolorosa y su alivio o exacerbación. También es importante someter a prueba los instrumentos existentes, aplicarlos y validarlos de acuerdo con las características de nuestra población, esto ayudaría a minimizar las dificultades metodológicas en cierta medida insalvables, que implica el evaluar una experiencia subjetiva, cuya medición depende necesariamente del reporte del sujeto.

$\mathrm{Al}$ igual que la evaluación, las intervenciones deben ser integrales para 
aumentar la efectividad del tratamiento, en ellas se debe incluir la participación activa del paciente, su familia, cuidador y demás personas que pueden constituir una red de apoyo importante.

La intervención psicológica, debe ser considerada parte del tratamiento integral del dolor, y su propósito fundamental debe ser incrementar la percepción de control del dolor por parte del paciente y modificar, en ciertos casos las creencias en torno a su condición y a su enfermedad, esto podría incidir favorablemente en la calidad de vida del paciente y su familia

Finalmente, existe la necesidad de seguir generando estudios sobre el dolor y su relación con factores psicológicos, tanto en su percepción, como en la respuesta y adherencia al tratamiento, dada la estrecha e indiscutible asociación entre ellos.

\section{REFERENCIAS BIBLIOGRÁFICAS}

Allende, S., y Lara, A. (2000). Manual de oncología. México: McGraw Hill.

Baron, M.A. (1993). Psicología de la salud: aplicaciones clínicas y estrategias de intervención. Madrid: Pirámide.

Bonica, J.J. (1983). Pain research and therapy: achievements of the past and challenges of the future. En J.J. Bonica y A. Lindblom (Eds.), Proceedings in Pain Research and Therapy (pp. 1-36). New York: Raven Press.

Brannon, L., y Feist, J. (2001). Psicología de la salud. México: International Thomson.

Ciaramella, A., y Paollo, P. (2001). Assessment of depression among cancer patients: the role of pain, cancer type and treatment. Psycho- Oncology, 10, 156-165.

Cipher, D., Fernández, E., y Cliford, A. (2001). Cost-effectiveness and health care utilization in a Multidisciplinary Pain Center: Comparison of three treatment groups. Journal of Clinical Psychology in Medical Setting, 8, 237-244.
Cruzado, J.A., y Labrador, F. (1991). Modificación de la conducta en pacientes con cáncer. Revista Española de Terapia del Comportamiento, 8, 225-257.

Eisenberg, M. (1994). Cognitive aspects of pain. En P.D. Wall y R. Melzack (Eds.), Textbook of pain (pp. 315-336). Edinburgh, Scotland: Churchill Livingston.

Gross, D., Fogg, L., Webster-Stratton, C., Garvey, Ch., Julion, W., y Grady, J. (2003). Parent Training of Toddlers in Day Care in LowIncome Urban Communities. Journal of Consulting and Clinical Psychology, 71, 261-278.

Hadedorrn, M., Sneeuw, K., y Aaronson, N. (2002). Changes in physical functioning and quality of life in patients with cancer response shift and relative evaluation of one's condition. Journal of Clinical Epidemiology, 55, 176-183.

Harkins, S.W., Price, D.D., y Braith, J. (1989). Effects of extraversion and neuroticism on experimental pain, clinical pain, and illness behavior. Pain, 36, 209-218.

Herrera, M., y Orazi, U. (2002). Does the neurologist contribute to the care of patients with chronic back pain? European Neurology, 48, 61-64.

Hwang, S., Chang, V., y Kasimis, B. (2002). Dynamic cancer pain management outcomes: the relationship between pain severity, pain relief, functional interference, satisfaction and global quality of life over time. Journal of Pain and Symptom Management, 23, 190-200.

Jacobsen, P.B., Andrykowski, M.A., y Thors, Ch. (2004). Relationship of catastrophizing to fatigue among women receiving treatment for breast cancer. Journal of Consulting \& Clinical Psychology. 72, 355-361.

Lee, Ch.T., Katz, J., Fearn, P.A., y Russo, P. (2002). Mode of presentation of renal cell carcinoma provides prognostic information. Urologic Oncology, 7, 135-136.

Maconi, G., Kurihara, H., Panizzo, V., Russo, A., Cristaldi, M., Marrelli, D., Roviello, F., Manzoni, G., Di Leo, A., Morgagni, P., Bechi, P., Porro, G.B., y Taschieri, A.M. (2003). Gastric cancer in young patients with no alarm symptoms: focus on delay in diagnosis, stage of neoplasm and survival. Scandinavian Journal of Gastroenterology, 38, 1249-1257. 
Porter, L., Keefe, F., McBride, C., Pollak, K., Fish, L., y Garst, J. (2002). Perceptions of patients self efficacy for managing pain and lung cancer symptoms: correspondence between patients and family caregivers. Pain, 1, 169-178.

Redinbaugh, E., Baum, A., DeMoss, C., Fello, M., y Arnold, R. (2002). Factors Associated with the accuracy of family caregivers estimates of patient pain. Journal of Pain and Symptom Management, 23, 31-38.

Schumacher, K., Koresawa, S., West, C., Hawkins, Ch., Johnson, C., Wais, E., Dodd, M., Paut, S., Triphathy, D., Koo, P., Phaim, D., y Miaskowski, Ch. (2002). Putting cancer pain management regimens into practice at home. Journal of Pain and Symptom Management, 23, 369-382.

Sela, R., Bruera, E., Conner-Spady, B., Cumming, C., y Walker, C. (2002). Sensory and affective dimensions of advanced cancer pain. Psycho-Oncology, 11, 23-24.

Simón, M.A. (1999). Manual de psicología de la salud: Fundamentos, metodología y aplicaciones. Madrid: Pirámide.

Stratton, J.H. (1996). Oncología Clínica. Manual de la American Cancer Society. México: OPS \& American Cancer Society.

Turk, D., y Melzack, R. (1992). Handbook of pain assessment. New York, USA: Guilford Press.

Willoughby, S.G., Hailey, B.J., Mulkana, Sh., y Rowe, J. (2002). The effect of laboratoryinduced depressed mood state on responses to pain. Behavioral Medicine, 28, 23-29.

Zaza, Ch., Reyno, L., y Moulin, D. (2000) The Multidimensional Pain Inventory profiles in patients with chronic cancer-related pain: an examination of generalizability. Pain, 87, 75-82. 